\title{
CERCLES DE REMPLISSAGE AND ASYMPTOTIC BEHAVIOUR
}

\author{
PAUL GAUTHIER
}

1. Introduction. In 1908 , Lindelöf showed that if $w=f(z)$ is a bounded holomorphic function in a sector $S$ : $|\arg z|<\theta_{1}$, and if $f(z)$ has an asymptotic value $w_{0}$ as $z$ tends to $\infty$ along a half-ray in $S$; then $f(z)$ tends uniformly to $w_{0}$ as $z$ tends to $\infty$ within any sector $|\arg z| \leqq \theta, 0 \leqq \theta<\theta_{1}$. Montel (8) later replaced the condition that $f(z)$ be bounded by the condition that $f(z)$ be meromorphic and omitted three values. The following is an immediate consequence of the Lindelöf-Montel theorem.

THEOREM 1. Let $w=f(z)$ be a function meromorphic in the sector $|\arg z|<\theta_{1}$, and let $f(z)$ tend to a value $w_{0}$ as $z \rightarrow \infty$ along the positive real axis. Then there is a number $\theta_{0}\left(0 \leqq \theta_{0} \leqq \theta_{1}\right)$ such that:

(1) If $0 \leqq \theta<\theta_{0}$, then $f(z) \rightarrow w_{0}$ uniformly in the sector $|\arg z| \leqq \theta$; and

(2) If $\theta_{0}<\theta_{1}$, then for each $\theta$ such that $\theta_{0}<\theta<\theta_{1}, f(z)$ assumes every value of the Riemann sphere, with at most two exceptions, infinitely often in the sector $|\arg z| \leqq \theta$.

This generalizes a theorem of Seidel (11, Corollary 5). An alternative formulation of the Lindelöf-Montel theorem which deals with strips rather than sectors obviously yields the following analogue to Theorem 1.

THEOREM 2. Let $w=f(z)$ be a function meromorphic in the strip $|\operatorname{Im} z|<R_{1}$, and let $f(z)$ tend to a value $w_{0}$ as $z \rightarrow \infty$ along the positive real axis. Then there is a number $R_{0}\left(0 \leqq R_{0} \leqq R_{1}\right)$ such that:

(1) If $0 \leqq R<R_{0}$, then $f(z) \rightarrow w_{0}$ uniformly in the strip $|\operatorname{Im} z| \leqq R$; and

(2) If $R_{0}<R_{1}$, then $f(z)$ assumes every value of the Riemann sphere, with at most two exceptions, infinitely often in each strip $|\operatorname{Im} z| \leqq R$, with $R_{0}<R<R_{1}$.

These two theorems are our main results, and the remainder of this paper will be devoted to generalizing these theorems in various directions. In order to sharpen part (2) in these theorems, we shall find it convenient to introduce (in $\S 2$ ) some notions adapted from Milloux's theory of cercles de remplissage.

2. Cercles de remplissage. Let $(S, s)$ be a metric space, where $S$ is a Riemann surface and $s$ is a metric for $S$. For a subset $A \subset S$ and a nonnegative number $r$, we write

$$
\Delta_{s}(A, r)=\{z \in S: s(A, z) \leqq r\} .
$$

Received November 30, 1967 and in revised form September 26, 1968. 
If the set $A$ is a singleton $A=\left\{z_{0}\right\}$, we write $\Delta_{s}\left(z_{0}, r\right)$ instead of $\Delta_{s}\left(\left\{z_{0}\right\}, r\right)$ and refer to $\Delta_{s}\left(z_{0}, r\right)$ as an $s$-disc. When no confusion is likely as to which metric is being employed, we write $\Delta(A, r)$ for $\Delta_{s}(A, r)$, and we shall also use the notation $\left\{\Delta_{n}\right\}$ to signify a sequence of discs.

Definition. Let $w=f(z)$ be a meromorphic function on the Riemann surface $(S, s)$. A sequence of points $\left\{z_{n}\right\}, z_{n} \in S$, is called a sequence of $s$-points for the function $f(z)$ if there are sequences $\left\{L_{n}\right\}$ and $\left\{r_{n}\right\}$, satisfying

(A) $L_{1}>L_{2}>\ldots>L_{n}>\ldots, L_{n} \rightarrow 0$, for $n \rightarrow \infty$,

(B) $r_{1}>r_{2}>\ldots>r_{n}>\ldots, r_{n} \rightarrow 0$, for $n \rightarrow \infty$, and

(C) in each disc $\Delta_{n}=\Delta_{s}\left(z_{n}, r_{n}\right), n=1,2, \ldots$,

the function $f(z)$ assumes all values of the Riemann sphere with the possible exception of two sets of values $E(n)$ and $G(n)$ whose spherical diameters do not exceed $L_{n}$.

The sequence $\left\{\Delta_{n}\right\}$ of discs is called a sequence of s-cercles de remplissage for $f(3)$.

Corollary 1. Let $w=f(z)$ be a meromorphic function on $(S, s)$. Let $\{z$ \} $\}$ be a sequence of s-points for $f(z)$, and let $\left\{z_{n}{ }^{\prime}\right\}, z_{n}{ }^{\prime} \in S$, be a sequence of points for which $s\left(z_{n}, z_{n}^{\prime}\right) \rightarrow 0$, as $n \rightarrow \infty$. Then $\left\{z_{n}^{\prime}\right\}$ is also a sequence of s-points.

If a family $F$ of functions meromorphic on the Riemann surface $(S, s)$ is not normal on $S$, then there is some point $z_{0} \in S$ at which the family is not normal. It follows (9, pp. 219, 220) that there is a sequence of functions $\left\{f_{n}\right\}, f_{n} \in F$, no subsequence of which converges in any neighbourhood of $z_{0}$. Such a sequence is said to be an exceptional sequence of meromorphic functions at the point $z_{0}$.

The following theorem, originally stated for families of functions meromorphic on a subset of the Riemann sphere, also holds, by an obvious modification of the original proof, for a family of functions meromorphic on a Riemann surface $S$.

THEOREM 3 (Ostrowski (9, p. 234)). If $\left\{f_{n}\right\}$ is an exceptional sequence of meromorphic functions at the point zo of the Riemann surface $S$ on which each function $f_{n}$ is defined, and if $\epsilon>0$ and $U$ is an arbitrary open set on $S$ about zo, then each of the functions $f_{n}$ for $n>n_{0}(\epsilon, U)$ assumes every value of the Riemann sphere in $U$, with the possible exception of two sets of values $E(\epsilon, U, n)$ and $G(\epsilon, U, n)$ whose spherical diameters do not exceed $\epsilon$.

CoROLlary 2. If $\left\{f_{n}\right\}$ is an exceptional sequence of meromorphic functions at the point zo of a Riemann surface $(S, s)$ on which each function $f_{n}$ is defined, then there are sequences $\left\{L_{n}\right\}$ and $\left\{r_{n}\right\}$, where

(A) $L_{1}>L_{2}>\ldots>L_{n}>\ldots, L_{n} \rightarrow 0$, for $n \rightarrow \infty$,

(B) $r_{1}>r_{2}>\ldots>r_{n}>\ldots, r_{n} \rightarrow 0$, for $n \rightarrow \infty$, and

(C) in each disc $\Delta_{s}\left(z_{0}, r_{n}\right), n=1,2, \ldots$, the function $f_{n}(z)$ assumes every value of the Riemann sphere with the possible exception of two sets of values $E(n)$ and $G(n)$ whose spherical diameters do not exceed $L_{n}$. 


\section{Functions allowing a meromorphic continuation throughout} the Euclidean plane punctured at the origin. For $p=1,2, \ldots$, let $S_{p}$ be the covering surface associated with the function $w=\exp (1 / p \log z)$, and let $S_{\infty}$ be the covering surface associated with $w=\log z$. For $p=1,2, \ldots, \infty$, we consider the origin to be deleted from $S_{p}$. In the following definitions, $p$ may be finite or infinite. Denote by $z(z)$ the projection of a point $z \in S_{p}$ into the plane. To any point $z \in S_{p}$ we can give coordinates $z=(r, \theta)$, where $r=|z(z)|$, and $\theta$ is a suitably chosen value of $\arg z(z)$ for which $-p \pi<\theta \leqq+p \pi$. Set $\arg _{p z}=\theta$ and $|z|=r$. For $z_{1}, z_{2} \in S_{p}$, with $z_{1}=\left(r_{1}, \theta_{1}\right)$, $z_{2}=\left(r_{2}, \theta_{2}\right)$, we define $z_{1} z_{2}=(r, \theta)$ by setting $r=r_{1} r_{2}$ and $\theta$ equal to that value in $-p \pi<\theta \leqq p \pi$ which is congruent to $\theta_{1}+\theta_{2}$ modulo $2 p \pi$. We define $z_{1} / z_{2}$ as $z_{1} z_{3}$, where $z_{3}=\left(1 / r_{2},-\theta_{2}\right)$. For $z \in S_{p}$, set $l_{p}(z)=\log |z|+i \arg p z$. Since $r$ and $|\theta|$ vary continuously with $z=(r, \theta)$, it can be verified that

$$
\sigma_{p}\left(z_{1}, z_{2}\right)=\left|l_{p}\left(z_{1} / z_{2}\right)\right|
$$

is a continuous metric for $S_{p}$. For a subset $A \subset S_{p}$ and a value $w_{0}$ of the Riemann sphere, we say that a function $f(z)$ defined on $S_{p}$ tends to $w_{0}$ in $A$ if $f\left(z_{n}\right) \rightarrow w_{0}$ for each sequence $\left\{z_{n}\right\}, z_{n} \in A$, for which $\left|z_{n}\right| \rightarrow \infty$. A boundary $\operatorname{arc} \alpha$ is a continuous curve $\alpha(t), 0 \leqq t<1$, on $S_{p}$ for which $|\alpha(t)| \rightarrow \infty$ as $t \rightarrow 1$.

We remark that the above definitions have been made for the purpose of studying the behaviour of functions near the point at infinity on $S_{p}$. Actually, the origin plays a role symmetric to that of the point at infinity, and we could just as easily study the behaviour of functions near the origin.

Suppose that $w=f(z)$ can be continued meromorphically throughout the plane punctured at the origin, and let $S$ be the covering surface for $f(z)$, with the origin deleted from $S$. Then for some $p=1,2, \ldots, \infty$, we have $S=S_{p}$. Set $\sigma=\sigma_{p}$. Then $f(z)$ induces a single-valued function $w=f(z)$ defined on the surface $(S, \sigma)$.

THEOREM 4. Let $w=f(z)$ allow a meromorphic continuation throughout the plane punctured at the origin, and let $w=f(z)$ be the induced meromorphic function on the covering surface $(S, \sigma)$. If $f(\xi)$ tends to a value wo along a boundary arc $\alpha$, then there is a number $\theta_{0}\left(0 \leqq \theta_{0} \leqq p \pi\right)$ such that:

(1) If $0 \leqq \theta<\theta_{0}$, then $f(z) \rightarrow w_{0}$ uniformly in the set $\Delta_{\sigma}(\alpha, \theta)$; and

(2) If $\theta_{0}<p \pi$, then there is a sequence of $\sigma$-cercles de remplissage whose respective centres lie on the boundary of $\Delta_{\sigma}\left(\alpha, \theta_{0}\right)$.

Proof. Let $F=\left\{f_{t}(\xi): 0 \leqq t<1\right\}$, where $f_{t}(\xi)=f\left(g_{t}(\xi)\right)$, with $g_{t}(\xi)=\alpha(t) \xi$. We denote by 1 the point $z=(1,0)$ in $S$.

Suppose that for some $\theta>0, F$ is normal in $\Delta(1, \theta)$. In this case let

$$
\theta_{0}=\operatorname{lub}_{0 \leqq \theta<p \pi}\{\theta: F \text { is normal in } \Delta(1, \theta)\} .
$$

Clearly then $\theta_{0}>0$. We now show that (1) is satisfied. Suppose that $0<\theta<\theta_{0}$ and $\left\{z_{n}\right\}$ is any sequence of points in $\Delta(\alpha, \theta)$ such that $\left|z_{n}\right| \rightarrow \infty$ as $n \rightarrow \infty$. 
Since $z_{n} \in \Delta(\alpha, \theta)$, there is a point $t_{n}, 0 \leqq t_{n}<1$, such that $z_{n} \in \Delta\left(\alpha\left(t_{n}\right), \theta\right)$, $n=1,2, \ldots$. Set $f_{n}=f_{t}$ and $g_{n}=g_{t}$ with $t=t_{n}$, and let $y_{n}$ be a point of $\Delta(1, \theta)$ for which $g_{n}\left(y_{n}\right)=z_{n}$. Then $f\left(z_{n}\right)=f_{n}\left(y_{n}\right)$. Since $F$ is a normal family in $\Delta\left(1, \theta_{0}\right),\left\{f_{n}\right\}$ has a subsequence $\left\{f_{n(k)}\right\}$ which converges spherically uniformly on $\Delta(1, \theta)$ to a function $g(z)$ which is meromorphic or identically infinite. We shall show that $g(z)$ is identically equal to $w_{0}$. Fix $R, 0 \leqq R \leqq \theta$, and for each $k$, let $a_{k}$ denote a point for which $g_{n(k)}\left(a_{k}\right)$ is on $\alpha$ and $\sigma\left(1, a_{k}\right)=R$. Let $z_{R}$ be a limit point of the sequence $\left\{a_{k}\right\}$. Since $\left\{f_{n(k)}(z)\right\}$ converges spherically uniformly to $g(z)$, and since $g(z)$ is spherically continuous, $f_{n(k)}\left(a_{k}\right) \rightarrow g\left(z_{R}\right)$. Since $f_{n(k)}\left(a_{k}\right)=f\left(g_{n(k)}\left(a_{k}\right)\right)$ and $g_{n(k)}\left(a_{k}\right) \rightarrow \infty$ along $\alpha$, it follows that $g(z R)=w_{0}$ since $f(z) \rightarrow w_{0}$ along $\alpha$. Hence, for each $R, 0 \leqq R \leqq \theta$, there is a point $z_{R}$, with $\sigma\left(1, z_{R}\right)=R$, such that $g\left(z_{R}\right)=w_{0}$, and therefore $g\left(z^{\prime}\right)$ is identically equal to $w_{0}$. It follows that $f_{n(k)}\left(y_{n(k)}\right) \rightarrow w_{0}$, as $k \rightarrow \infty$. However, $f_{n(k)}\left(y_{n(k)}\right)=f\left(z_{n(k)}\right)$, and $\left\{z_{n}\right\}$ was an arbitrary sequence of points in $\Delta(\alpha, \theta)$ for which $z_{n} \rightarrow \infty$. We have shown that any such sequence has a subsequence on which $f(z)$ tends to $w_{0}$. It follows that $f(z) \rightarrow w_{0}$ uniformly in $\Delta(\alpha, \theta)$. This verifies (1) in the case where $F$ is normal in $\Delta(1, \theta)$ for some $\theta>0$.

Suppose, on the other hand, that, for each $\theta>0, F$ is not normal in $\Delta(1, \theta)$. Then, if we set $\theta_{0}=0 ;(1)$ is vacuous, and therefore (1) holds in all cases.

To prove (2), suppose that $\theta_{0}<p \pi$. Then there is a point $z_{0}$ on the boundary of $\Delta\left(1, \theta_{0}\right)$ such that $F$ is not normal at $z_{0}$. Let $\left\{f_{n}\right\}, f_{n} \in F$, be an exceptional sequence at the point $z_{0}$ and let $t_{n}$ be such that $f_{n}=f_{t}$, for $t=t_{n}$. Set $g_{n}=g_{t}$, for $t=t_{n}$. Since the functions $g_{n}, n=1,2, \ldots$, are $\sigma$-isometries, it follows from Corollary 2 that $\left\{g_{n}\left(z_{0}\right)\right\}$ is a sequence of $\sigma$-points for $f(z)$ which lie in $\Delta\left(\alpha, \theta_{0}\right)$. By part (1) of Theorem 4 , for each $0<\theta<\theta_{0},\left\{g_{n}\left(z_{0}\right)\right\}$ is eventually outside of $\Delta(\alpha, \theta)$, and hence there is a sequence $\left\{z_{n}\right\}$ of points on the boundary of $\Delta\left(\alpha, \theta_{0}\right)$ such that $\sigma\left(z_{n}, g_{n}\left(z_{0}\right)\right) \rightarrow 0$. From Corollary 1 it follows that $\left\{z_{n}\right\}$ is also a sequence of $\sigma$-points and the proof is complete.

We remark that the above theorem can be generalized to functions which allow a meromorphic continuation throughout a punctured neighbourhood of some point.

Let $w=f(z)$ be a function meromorphic in the Euclidean plane, and let $d$ denote the Euclidean metric. The function $f(z)$ is said to have a sequence of cercles de remplissage in the sense of Milloux provided there is a sequence of $d$-discs $\left\{\Delta_{n}\right\}$ such that the angle subtended by $\Delta_{n}$ as seen from the origin shrinks to zero as $n$ increases, and in each $\Delta_{n}$ the function $f(z)$ assumes every value of the Riemann sphere with the possible exception of two sets of values $E(n)$ and $G(n)$ whose spherical diameters shrink to 0 as $n$ increases.

Lemma 1. Let $w=f(z)$ be a function meromorphic in the Euclidean plane. Then $f(z)$ has a sequence of $\sigma$-cercles de remplissage if and only if it has a sequence of cercles de remplissage in the sense of Milloux.

Proof. Suppose that $\left\{\Delta_{\boldsymbol{\sigma}}\left(z_{n}, \phi_{n}\right)\right\}$ is a sequence of $\sigma$-cercles de remplissage for 
$f(z)$. Let $\psi_{n}$ be such that $\sin \psi_{n}=\exp \left(\phi_{n}\right)-1$. For sufficiently large $n, \psi_{n}$ can be found and $\phi_{n}<\psi_{n}$. For $z \in \Delta_{\sigma}\left(1, \phi_{n}\right)$,

Hence,

$$
|z-1| \leqq \exp \left(\phi_{n}\right)-1 \text {. }
$$

$$
\Delta_{\sigma}\left(1, \phi_{n}\right) \subset \Delta_{d}\left(1, R_{n}\right),
$$

where $R_{n}=\exp \left(\phi_{n}\right)-1$. Let $\Delta_{n}=z_{n} \Delta_{d}\left(1, R_{n}\right)$. Then $\left\{\Delta_{n}\right\}$ is a sequence of cercles de remplissage in the sense of Milloux.

Conversely, let $\left\{\Delta_{n}\right\}$ be a sequence of cercles de remplissage in the sense of Milloux. Choose $z_{n}$ and $R_{n}$ such that $\Delta_{n}=z_{n} \Delta_{d}\left(1, R_{n}\right)$. Let

$$
\psi_{n}=\sup \left\{\sigma(1, z): z \in \Delta_{d}\left(1, R_{n}\right)\right\} .
$$

Then $\psi_{n} \rightarrow 0$ as $n \rightarrow \infty$, and

$$
\Delta_{d}\left(1, R_{n}\right) \subset \Delta_{\sigma}\left(1, \psi_{n}\right) .
$$

Multiplying both sides by $z_{n}$ yields

$$
\Delta_{n} \subset \Delta_{\sigma}\left(z_{n}, \psi_{n}\right) \text {. }
$$

Thus, $\left\{\Delta_{\sigma}\left(z_{n}, \psi_{n}\right)\right\}$ is a sequence of $\sigma$-cercles de remplissage.

We are now in a position to prove the following generalization of Julia's theorem.

Milloux's THEOREM (7). Let $w=f(z)$ be a function meromorphic in the Euclidean plane with an essential singularity at $\infty$, and let $f(z)$ have an asymptotic value at $\infty$. Then $f(z)$ has a sequence of cercles de remplissage in the sense of Milloux.

Proof. By Lemma 1 it is enough to show that $f(z)$ has a sequence of $\sigma$-cercles de remplissage. Let $\alpha$ be the asymptotic path and $w_{0}$ the asymptotic value for $f(z)$ at $\infty$. Denote by $\theta_{0}=\theta(\alpha)$ the critical number whose existence is asserted in Theorem 4. Then $0 \leqq \theta(\alpha) \leqq \pi$.

Case 1. $\theta(\alpha)<\pi$. Then by Theorem 4 there is a sequence of $\sigma$-cercles de remplissage for $f(z)$.

Case 2. $\theta(\alpha)=\pi$. Set $\alpha^{\prime}=\left\{z=\left(r, \theta+\frac{1}{2} \pi\right):(r, \theta) \in \alpha\right\}$. Then $\alpha^{\prime} \subset \Delta_{\sigma}\left(\alpha, \frac{1}{2} \pi\right)$, and therefore by Theorem $4, f(z) \rightarrow w_{0}$ on $\alpha^{\prime}$. Applying Theorem 4 to $\alpha^{\prime}$ we obtain a critical number $\theta\left(\alpha^{\prime}\right)$. If $\theta\left(\alpha^{\prime}\right)=\pi$, then since $\theta(\alpha)=\pi$, it follows from (1) of Theorem 4 that $f(z) \rightarrow w_{0}$ uniformly as $z \rightarrow \infty$. This contradicts the fact that $\infty$ is an essential singularity. Hence, $\theta\left(\alpha^{\prime}\right)<\pi$, and we are back to Case 1. This concludes the proof.

The above is a weak form of Milloux's theorem. The complete theorem actually gives an estimate on the various diameters. Further contributions in this direction were made by Valiron (14). 
Let $w=f(z)$ be a function meromorphic in the Euclidean plane. We shall say that $f$ is a Milloux exceptional function if $f$ has no sequence of cercles de remplissage in the sense of Milloux. The function $f$ is said to be a Julia exceptional function if the family $\left\{f_{t}: 0<|t|<\infty\right\}$ is normal in $0<|z|<\infty$, where $f_{t}(z)=f(t z)$. Both Marty (6) and Ostrowski (9) have characterized the Julia exceptional functions. In fact, Ostrowski gave a representation theorem for such functions (9, Theorem 5). From Corollary 2 and Lemma 1, it follows that the Julia exceptional functions are precisely the Milloux exceptional functions. Both the characterizations of Marty and Ostrowski then apply to the Milloux exceptional functions.

4. Functions meromorphic in the neighbourhood of an essential singularity. Let $w=f(z)$ be a function meromorphic in a neighbourhood $S_{0}$ of an essential singularity $z_{0}$. Let $h(z)$ denote the rotation of the Riemann sphere which carries the point $z_{0}$ to the point at infinity. For any two points $z_{1}, z_{2} \in S_{0}$, we write

$$
d\left(z_{1}, z_{2}\right)=\left|h\left(z_{1}\right)-h\left(z_{2}\right)\right| .
$$

Then $\left(S_{0}, d\right)$ is a metric space. For a subset $A \subset S_{0}$ and a value $w_{0}$ of the Riemann sphere, we say that the function $f(z)$ tends to $w_{0}$ in $A$ if $f\left(z_{n}\right) \rightarrow w_{0}$ for each sequence $\left\{z_{n}\right\}, z_{n} \in A$, for which $z_{n} \rightarrow z_{0}$. A boundary arc $\alpha$ is a continuous curve $\alpha(t), 0 \leqq t<1$, on $S_{0}$ for which $\alpha(t) \rightarrow z_{0}$ as $t \rightarrow 1$.

THEOREM 5. Let $w=f(z)$ be a function meromorphic in a (deleted) neighbourhood $S_{0}$ of an essential singularity $z_{0}$, and let $f(z)$ have an asymptotic value $w_{0}$ along a boundary arc $\alpha$. Then there is a number $R(\alpha)(0 \leqq R(\alpha) \leqq+\infty)$ such that

(1) If $0 \leqq R<R(\alpha)$, then $f(z) \rightarrow w_{0}$ uniformly in the set $\Delta_{d}(\alpha, R)$; and

(2) If $R(\alpha)<+\infty$, then there is a sequence of $d$-cercles de remplissage whose centres lie on the boundary of $\Delta_{d}(\alpha, R(\alpha))$.

Proof. The proof is analogous to the proof of Theorem 4. One redefines $g_{t}(z)$ as

$$
g_{t}(z)=h^{-1}(h(\alpha(t))+z) .
$$

Then one considers the behaviour of the family $F$ in neighbourhoods $\{z:|z| \leqq R\}$ of the origin. The details are omitted.

Consider the special case that $z_{0}$ is the point at infinity and $f(z)$ is meromorphic in the Euclidean plane. Then $S_{0}=(|z|<\infty)$ and the metric $d$ is just the Euclidean metric. From this it is seen that a sequence of $d$-cercles de remplissage is a fortiori a sequence of cercles de remplissage in the sense of Milloux. It is further seen that a function $f(z)$ meromorphic in the Euclidean plane has a sequence of $d$-cercles de remplissage if and only if the family $\left\{f_{t}(z):|t|<\infty\right\}, f_{t}(z)=f(z+t)$, is not normal in $|z|<\infty$. Functions $f(z)$ for which the family $\left\{f_{t}(z):|t|<\infty\right\}$ is not normal have been studied by Yosida (15). 
5. Examples. Let $w=f(z)$ be a function meromorphic in the Euclidean plane which has an asymptotic value along an $\operatorname{arc} \alpha$, and let $\theta(\alpha), R(\alpha)$ be the numbers whose existences are asserted by Theorems 4 and 5 , respectively. We remark that if $\theta(\alpha)>0$, then $R(\alpha)=+\infty$.

Example 1. $f(z)$ entire transcendental; $\theta(\alpha)=\pi$. There exists an entire transcendental function $w=f(z)$ which tends to 0 uniformly as $z \rightarrow \infty$ in an angle inferior to $2 \pi$ by as little as one pleases. This result is due to MittagLeffler; see (5, p. 120). [I am grateful to W. Fuchs for this reference.]

Example 2. $\theta(\alpha)=0, R(\alpha)=+\infty$. Let $f(z)=z e^{z}$ and let $\alpha$ be the positive imaginary axis. Then it is easily seen that $\theta(\alpha)=0$, but $R(\alpha)=+\infty$. [I am indebted to K.-F. Tse for this example.]

Example 3. $\theta(\alpha)=0,0<R(\alpha)<+\infty$. Let $f(z)=\exp \left(e^{z}\right)$, and let $\alpha$ be the positive real axis. Then one can verify that $\theta(\alpha)=0$, and that $R(\alpha)=\frac{1}{2} \pi$. The calculations are omitted.

Example 4. $\theta(\alpha)=0, R(\alpha)=0$. Let $\alpha$ be the positive real axis, and let $\alpha^{\prime}(t)=1 / t, 1 \leqq t<+\infty$. By a theorem of Bagemihl and Seidel $(1$, Theorem 5$)$, there exists an entire function $w=f(z)$ which tends to 0 and 1 on $\alpha$ and $\alpha^{\prime}$, respectively. From the above construction, it follows that $\theta(\alpha)=0$ and $R(\alpha)=0$.

In the next example, $\theta(\alpha)=+\infty$. The function $f(z)=\log z$ has this property, but this is a trivial example since $\log z \rightarrow \infty$ uniformly as $z \rightarrow \infty$.

Example 5. There exists a function $f(z)$ having distinct asymptotic values on two asymptotic paths $\alpha$ and $\alpha^{\prime}$ for which $\theta(\alpha)=+\infty$ and $\theta\left(\alpha^{\prime}\right)=+\infty$.

Proof. Let $f(z)=z^{i+1}$, and let $\alpha$ be the positive real axis. Suppose that $z \in \Delta_{\sigma}(\alpha, \theta)$ for some $\theta<+\infty$. Then $|f(z)|=|z \exp (-\arg z+i \log |z|)|=$ $|z| \exp (-\arg z) \geqq e^{-\theta}$. Thus, $f(z) \rightarrow \infty$ as $z \rightarrow \infty$ in $\Delta(\alpha, \theta)$, and the same argument works for any finite $\theta$. Hence $\theta(\alpha)=+\infty$.

Let $\alpha^{\prime}(t)=t e^{i t}, 0 \leqq t<+\infty$. Then $z \rightarrow \infty$ on $\alpha^{\prime},\left|f\left(t e^{i t}\right)\right|=t e^{-t}$. Hence, $f(z) \rightarrow 0$ on $\alpha^{\prime}$. Let $\theta>0$, and suppose that $z \in \Delta\left(\alpha^{\prime}, \theta\right)$. Then for $|z|=r$,

$$
r-\theta \leqq \arg z \leqq r+\theta
$$

and therefore

$$
|f(z)| \leqq r e^{-r+\theta} .
$$

Thus, $f(z) \rightarrow 0$ in $\Delta\left(\alpha^{\prime}, \theta\right)$, and thus $\theta\left(\alpha^{\prime}\right)=+\infty$.

6. Functions meromorphic in the unit disc. In this section we consider the space $(D, \rho)$, where $D$ is the unit disc $|z|<1$ and $\rho$ is the nonEuclidean hyperbolic metric on $D$. The notion of a sequence of $\rho$-cercles $d e$ remplissage was introduced by Lange (4). 
For a subset $A \subset D$ and a value $w_{0}$ of the Riemann sphere, we say that a function $f(z)$ defined on $D$ tends to $w_{0}$ in $A$ if $f\left(z_{n}\right) \rightarrow w_{0}$ for each sequence $\left\{z_{n}\right\}, z_{n} \in A$, for which $\left|z_{n}\right| \rightarrow 1$. A boundary arc $\alpha$ is a continuous curve $\alpha(t), 0 \leqq t<1$, on $D$ for which $|\alpha(t)| \rightarrow 1$ as $t \rightarrow 1$.

THEOREM 6. Let $w=f(z)$ be a function meromorphic in $D$ which tends to $a$ value $w_{0}$ along a boundary arc $\alpha$ in $D$. Then there is a number $r(\alpha)$ $(0 \leqq r(\alpha) \leqq+\infty)$ such that:

(1) If $0 \leqq r<r(\alpha)$, then $f(z) \rightarrow w_{0}$ uniformly in the set $\Delta_{\rho}(\alpha, r)$; and

(2) If $r(\alpha)<+\infty$, then there is a sequence of $\rho$-cercles de remplissage whose respective centres lie on the boundary of $\Delta_{\rho}(\alpha, r(\alpha))$.

Proof. In the proof of Theorem 4 we replace $g_{t}(z)$ by

$$
g_{t}(z)=[z+\alpha(t)] /[1+\overline{\alpha(t)} z]
$$

and we consider the behaviour of the family $F$ in neighbourhoods $\Delta_{\rho}(0, r)$ of the origin. Since the remainder of the proof is analogous, the details are omitted.

The above theorem concerning functions meromorphic in a disc can be generalized to functions meromorphic in a region whose boundary contains an analytic arc. Theorem 6, which generalizes a result of Gavrilov (3, Theorem 4) and bears some resemblance to a result of Stebbins (12), formed part of my doctoral dissertation written under Professor W. Seidel. For applications of Theorem 6 , see $(\mathbf{1 0} ; \mathbf{1 3} ; \mathbf{2})$.

\section{REFERENCES}

1. F. Bagemihl and W. Seidel, Some boundary properties of analytic functions, Math. Z. 61 (1954), 186-199.

2. P. Gauthier, Cercles de remplissage and asymptotic behaviour along circuitous paths (to appear in Can. J. Math.).

3. V. I. Gavrilov, On a theorem of A. L. Shaginjan, Vestnik Moskov. Univ. Ser. I Mat. Meh., no. 2 (1966), 3-10. (Russian)

4. L. H. Lange, Sur les cercles de remplissage non-Euclidiens, Ann. Sci. École Norm. Sup. (3) 77 (1960), 257-280.

5. E. Lindelöf, Calcul des résidus (Chelsea, New York, 1947).

6. F. Marty, Recherches sur la répartition des valeurs d'une fonction méromorphe, Ann. Fac. Sci. Univ. Toulouse (3) 23 (1931), 183-261.

7. H. Milloux, Sur le théorème de Picard, Bull. Soc. Math. France 53 (1925), 181-207.

8. P. Montel, Sur les familles de fonctions analytiques qui admettent des valeurs exceptionnelles dans un domaine, Ann. Sci. École Norm. Sup. (3) 29 (1912), 487-535.

9. A. Ostrowski, Über Folgen analytischer Funktionen und einige Verschärfungen des Picardschen Satzes, Math. Z. 24 (1925), 215-258.

10. D. C. Rung, Behavior of holomorphic functions in the unit disk on arcs of positive hyperbolic diameter (to appear in J. Math. Kyoto Univ.).

11. W. Seidel, Holomorphic functions with spiral asymptotic values, Nagoya Math. J. 14 (1959), 159-171. 
12. J. Stebbins, Spiral asymptotic values of functions meromorphic in the unit disk, Nagoya Math. J. 30 (1967), 247-262.

13. K.-F. Tse, Some results on value distribution of meromorphic functions in the unit disk, Nagoya Math. J. (to appear).

14. G. Valiron, Compléments au théorème de Picard-Julia, Bull. Sci. Math. 51 (1927), 167-183.

15. K. Yosida, On a class of meromorphic functions, Proc. Phys.-Math. Soc. Japan (3) 16 (1934), 227-235.

Université de Montréal,

Montréal, Québec 